\title{
DEPARTMENT OF ERRORS
}

When we published the article

"Green, DA et al. (2006) Tachypnea and hypocapnia are induced by 'buffeting' in vehicles. Clin Auton Res 16(4):281-285" [The online version of the original article can be found at http://dx.doi.org/10.1007/s10286006-0360-5]

the key word "automatic nervous system" should have appeared as "autonomic nervous system".

The publisher apologies for any inconvenience that this may have caused. 